\title{
Modification of humic substances of coal by ferrocene
}

Zimbovskaya M.M., Reshetova M.D., Konstantinov A.I., Shatalova T.B., Farat O.K., Pankratov D.A.

Lomonosov Moscow State University, Moscow, Russia, pankratov@radio.chem.msu.ru

Keywords: ferrocene, chemical modification

doi: 10.36291/HIT.2019.zimbovskaya.100

The high reactivity of humic substances (HS) is caused by the presence of a large number of functional groups in the composition of their macromolecules: primarily carboxyl, hydroxyl, carbonyl, nitrogen and sulfur containing groups. Functional groups of HS are inclined towards selective coordination, especially in relation to transition metals. Several methods are known for the synthesis of preparations of HS and iron: by the interaction of aqueous solutions of HS and iron salts; by means of mechanical dispersion (dry grinding of HS powder and magnetite in a ball mill); by chemical coprecipitation in the presence of HS (the Massart method), and by the interaction of metallic iron with aqueous HS solutions by oxidative in situ synthesis [1]. However, there is no direct evidence of the existence in these compounds of coordination bonds between iron ions and functional groups of HS. Perhaps this is due to the limited number of accessible functional groups of HS capable of forming strong covalent bonds with iron ions, as well as steric hindrances. It is obvious that the modification of HS using ligands that are prone to the formation of coordination bonds with iron will make it possible to obtain HS compounds with a high content of fixed iron. This may be of practical interest, for example, for agrochemical purposes. Such a modification of the HS is possible as a result of the esterification of the cyclopentadienyl derivative with the hydroxyl groups of the HS. It is known that $\mathrm{C}_{5} \mathrm{H}_{6}$ easily forms cyclopentadienyl m-complexes with iron - as ferrocene.

Ferrocene has great sustainability, chemical and thermal stability, lability in redox processes, has non-toxic effects. Ferrocene can be used as initial compound for number of derivatives with various substituents. Therefore, ferrocene carboxylic acid chloride was used as the initial reagent for the modification of HS. The initial ferrocene was acylated with acetic anhydride for obtain acetylferrocene. Then ferrocene carboxylic acid was obtained by oxidation of acetylferrocene. The ferrocene carboxylic acid chloride was obtained at interaction of ferrocene carboxylic acid with thionyl chloride.

Modification of HS of coal by hydroxyl groups with ferrocene carboxylic acid chloride was carried out with benzoyl chloride under the conditions of inverse phase-transfer catalysis [2]. The product synthesized in the work is insoluble in water, but it is well soluble in some organic solvents. HS modified by ferrocene obtained as a result of described operations was characterized by such physico-chemical methods as: Mössbauer and IR spectroscopy, DTA, TGA with analysis of evolved gases and size-exclusion chromatography. It has been shown that the physico-chemical properties of the resulting HS modified by ferrocene contain characteristic features of both $\mathrm{HS}$ and ferrocene. Average molecular weight of HS after modification has decreased by half. Iron in the preparation is in three coordination positions, and $7 \%$ of iron (II) in one of them. In the samples is not confirmed present initial ferrocene or it derivatives used in the synthesis. Thus, the work carried out the modification of the HS by ferrocene and showed that the obtained compound has unique properties that differ from the initial HS.

The authors thank Borisova N.E for assistance in obtaining IR data and useful tips.

References

1. Pankratov D.A., Anuchina M.M. // Materials Chemistry and Physics. 2019. 231:216-224.

2. Khil'ko S.L. et al. // Solid Fuel Chemistry. 2015. 49(4):206-212. 